\title{
Papillary Pathology in Calcium Oxalate Stone Formers Who Do and Do Not Form Their Stones on Randall's Plaque
}

\author{
Haider N. Al-Awadi, BS ${ }^{1}$, \& James C. Williams, Jr., PhD²
}

1. Indiana University School of Medicine 2. Indiana University School of Medicine, Department of Anatomy, Cell Biology \& Physiology

\section{Background and Hypothesis:}

Nephrolithiasis currently affects about one-in-eleven people with a recurrence in up to half of those individuals. Formation of calcium oxalate $(\mathrm{CaOx})$ stone is most common. Some $\mathrm{CaOx}$ stones are known to form on Randall's plaque, which is a calcification of the renal papilla, and this kind of stone can be identified by morphology. We divided CaOx stone formers into two groups: Randall's plaque (RP) stone formers (RPSF) and non-Randall's plaque stone formers (NRPSF). We hypothesized that renal papillary pathologies would be different between these two groups of stone formers.

\section{Experimental Design or Project Methods:}

Surgical videos were assessed for papillary pathology using a semiquantitative grading system to measure papillary appearance in terms of ductal plugging and dilation, tissue surface pitting, loss of papillary contour, and RP. The second measure computed the papillary percent surface area of Randall's plaque and ductal plugging using still images of the papilla. The scoring and quantitative measures of the papillae were compared between the two patient groups. All work was done in a manner blinded to the patient group.

\section{Results:}

Two-tailed t-test showed that RPSF group had higher scores of pitting and RP and lower scores for plugging when compared to the NRPSF group. Similarly, the quantitative data showed that RPSF group had a lower percentage of plugging surface area and higher percentage of RP surface area.

\section{Conclusion and Potential Impact:}

These data show that persons forming their $\mathrm{CaOx}$ stones primarily on RP have a papillary pathology that differs from $\mathrm{CaOx}$ stone formers who make their stones by other mechanisms. RPSF have more RP and less ductal plugging. Since the underlying pathologies existing in RP stone formation are different from other $\mathrm{CaOx}$ stone formers, it is possible that certain treatments could be especially effective for this group, and thus these results suggest that clinical trials that separate out this group of $\mathrm{CaOx}$ stone formers are warranted. 